\title{
Coronary Artery Stenosis
}

National Cancer Institute

\section{Source}

National Cancer Institute. Coronary Artery Stenosis. NCI Thesaurus. Code C80427.

Narrowing of the coronary artery lumen diameter. 